\title{
Disclosure of Duchenne muscular dystrophy after newborn screening
}

\author{
Evelyn Parsons, Don Bradley, Angus Clarke
}

Breaking the news about an untreatable disease is always difficult. Every professional involved in this activity will be aware of shortcomings in their practice, but it is possible to develop better practices. We report on a protocol developed during the first four years of the evaluation of a newborn screening programme. Although there may be additional constraints in other areas of medical practice, the underlying principles could be more generally applied.

Newborn screening for Duchenne muscular dystrophy (DMD) was introduced into Wales in $1990 .{ }^{1} \mathrm{DMD}$ is a sex linked disease causing progressive muscle wasting. Boys often show signs of muscle deterioration around 3 to 4 years; they are wheelchair bound by 8-12 years, and most boys die in their second or third decade.

Newborn screening has been possible since 1975 , but has not been widely practised because the disease is untreatable, and there has been uncertainty about the effects on the family of such an early diagnosis. The potential benefits include the avoidance of distressing diagnostic delays, enabling the family to plan for the future in practical ways, including choice in future pregnancies, and the offer of physiotherapy at an early stage.

The research team, aware that newborn screening for DMD did not fit the traditional criteria for screening, introduced the programme as an 'opt-in' test on the basis of informed parental consent. Within two to three days of the delivery, the parents of newborn boys are given an information leaflet about the newborn screening programme for DMD. This emphasises the optional nature of this test because the condition is serious and incurable. The timing of disclosure of the results to families is not routinely mentioned to families at this stage. In addition, a psychosocial evaluation was set up from the start of the programme to assess the impact of screening on families, and a protocol was drawn up for health professionals with guidance for handling a positive result (see table 1 ).

Medical Genetics,

University ofWales

Collersity or Wales

College of Medicine,

Heath Park, Cardiff

CF4 4XW

E Parsons

D Bradley

A Clarke

Correspondence to:

Dr Clarke.

\section{A protocol to maximise parental choice and minimise distress}

The medical profession has traditionally carried the main responsibility for imparting diagnostic information to patients. It is an element in their practice that can be strategically planned or dealt with on an ad hoc basis. Research has highlighted two relevant issues. First, that the initial emotional trauma surrounding a disclosure has more impact on family stability than the burden of caring for a chronically sick child. ${ }^{2}$ Second, that by adopting a specific procedure parental dissatisfaction with disclosure can be avoided. ${ }^{3}$

One review of the literature identifies eight principles that the majority of parents support: (i) to be told together; (ii) to be told as soon as possible; (iii) to be told in a private place without interruptions; (iv) to be told without an audience of staff; (v) to be told honestly and directly by someone who conveys warmth and understanding; (vi) to be allowed privacy together after the initial interview; (vii) to be given the chance to meet a professional on a number of subsequent occasions to ask questions; and (viii) to be given time and space to consider their feelings. ${ }^{4}$ The recommendations of Scope are very similar. ${ }^{5}$

A diagnosis of DMD will cause distress whenever it is made, but in designing the protocol we aimed to minimise that distress by adopting a planned, strategic approach to making the disclosure. We have identified three distinct phases in making the disclosure: the preliminary groundwork, the main encounter(s), and the follow up.

PHASE 1: PRELIMINARY GROUNDWORK

The time available for setting up the encounter with the family will vary with the medical condition. In the case of newborn screening for DMD there is no clinical urgency. About $50 \%$ of the cases with initially raised serum creatine kinase will have a persistently raised creatine kinase, and most of these will have DMD. The others, with a transient increase of creatine kinase, which is normal at retest, are reassured that they do not have DMD or any other muscle disease. The medical (diagnostic) agenda is therefore to obtain a venous blood sample for a repeat creatine kinase test from any child with an initially raised value. This clinically driven agenda has to be negotiated with the primary health care team and their perception of the family situation.

\section{Liaison with the primary health care team}

When the screen positive baby is between 4-6 weeks old a meeting is arranged between the newborn screening coordinator and the fami- 
Table 1 Model for structured disclosure

\begin{tabular}{|c|c|}
\hline Phase & Action and goals \\
\hline 1. Preliminary groundwork & $\begin{array}{l}\text { Liason with primary health care team: to establish positive relationship with professionals in } \\
\text { personal contact with family } \\
\text { Meeting with nominated local paediatrician: to ensure that clinician is familiar with the protocol, } \\
\text { and that (s)he is given details about the family }\end{array}$ \\
\hline \multicolumn{2}{|c|}{ Key issue: Maximise primary health care team involvement } \\
\hline $\begin{array}{l}\text { Minimise family dis } \\
\text { 2. The main encounter }\end{array}$ & $\begin{array}{l}\text { Inform families of their situation } \\
\text { Take venous sample for creatine kinase } \\
\text { Give results to family }\end{array}$ \\
\hline \multicolumn{2}{|c|}{ Key issues: Maximise information and choices for families } \\
\hline $\begin{array}{l}\text { 3. Follow up } \\
\text { Key issue: Provide on-going }\end{array}$ & $\begin{array}{l}\text { Identification of key worker at primary health care level } \\
\text { ort that is family specific }\end{array}$ \\
\hline
\end{tabular}

ly's primary health care team (usually the general practitioner and health visitor). At the beginning of the programme contact took place during week 2 or 3 of a baby's life. As a result of feedback from families and health visitors this practice has been modified; the earliest age at which a query is now raised with a family is 6 weeks, and may be deferred until later if there is evidence of maternal depression or social isolation. This meeting for liaison has five specific objectives:

(1) To meet the primary health care team face to face.

(2) To inform the primary health care team about the screening programme and the protocol to be followed, to give them details about the disease and the resources they can utilise in terms of on-going support for the family.

(3) To review the family situation and establish whether it is the right time to raise the query. The family situation is the key element determining the timing of the venous blood test.

(4) To plan provisional dates when the blood can be taken by the paediatrician. It is important to ensure that the health visitor is available to accompany the family to the hospital and for subsequent support. This may require deferring the process for one to two weeks.

(5) To plan raising the query with the family, when, and by whom. The timing is critical- it needs to be when someone is available to be with the mother afterwards, and close enough to the provisional hospital appointment to avoid a long period of anxiety and yet not so close that the parents are unable to go together. The health visitor usually visits the family late in the afternoon of the day before the hospital appointment.

\section{Meeting with the nominated paediatrician}

To achieve consistency of practice throughout Wales, a network of paediatricians, nominated by their colleagues, has been established. They act as the key clinician for cases in their area. The meeting has three aims: (i) to discuss specific details of the protocol; (ii) to provide the paediatrician with background information on the family; and (iii) to arrange a time when the venous sample can be taken.

Four factors constrain the timing of the appointment at which the family will meet the paediatrician. It must be: (i) when the paediatrician can give the family time; (ii) when the health visitor is available to accompany them; (iii) when the local biochemistry laboratory can test the blood and give a result rapidly (usually within hours, certainly within 24 hours); and (iv) when the paediatrician can offer an unhurried follow up appointment (within 24 hours) to discuss the results.

In practice the blood is usually taken on a Tuesday or Wednesday, enabling the query to be raised with the family on the previous day, and the blood sample to be taken, the results to be given and on-going support to be made available during the same working week.

There are several advantages to following this protocol:

(1) The procedure is sensitive and responsive to each individual family situation.

(2) Parental distress is reduced because delays are minimised between the time when the first query is raised, the second blood sample is taken and the results are given.

(3) It familiarises the primary health care team with the screening programme and the disease, and it establishes an early liaison between health professionals in the community and the specialist genetics service.

(4) It recognises the importance of a mother not being alone when potentially distressing news is given.

(5) It encourages the involvement of the health visitor from the outset, emphasising the importance of their role as a 'family advocate'.

(6) It focuses attention to the sensitive nature of a presymptomatic disclosure of DMD, and helps the paediatrician to arrange an appointment when time can be given to the family.

PHASE 2: THE MAIN ENCOUNTERS-ESTABLISHING A DIAGNOSIS OF DMD

Stage 1: the venous sample

The initial encounter between the paediatrician and the family has seven main objectives:

(1) To ensure that the family know which test is being queried. Newborn screening for DMD is optional, so all parents should be aware of the choice they have made. Research has shown, however, that parents have a low level of awareness about the diseases for which their baby has been tested. ${ }^{6-8}$

(2) To explain the difference between the initial screening test and the venous test, and to ensure that the family understands that transient increases can occur.

(3) To request the family's informed consent to the venous sample. 
(4) To give the family time to ask questions and to talk about their feelings.

(5) To discuss with the family how they would like to hear the results (that is, do they want to wait at the hospital, come back for another appointment, or see the paediatrician at home). This element in the protocol is left flexible for negotiation between paediatrician, health visitor, and family. Only two guidelines are given: first, in the case of a transient increase contact should be made as soon as possible either face to face or by telephone. Second, in all cases results should be given within 24 hours.

(6) To request the family's informed consent to participating in the social evaluation of the newborn screening programme.

(7) To initiate a positive relationship between the paediatrician and both the family and the health visitor.

\section{Stage 2: the results given}

A positive test result is always given to a family face to face by the paediatrician, preferably with the health visitor present. Whether that encounter takes place in the family home or hospital is negotiated in advance between those directly concerned. The protocol stresses, however, that time is set aside by the paediatrician so that the situation can be fully discussed. The main objectives of the encounter are:

(1) To inform the family of the results.

(2) To explain that a persistent increase in the creatine kinase value, although indicative of $\mathrm{DMD}$, is not diagnostic. This allows the family to consider how they would like further investigations to be paced. Do they want DNA (molecular genetic) tests at once? Do they want to proceed to muscle biopsy? If so, when?

In two thirds of cases of DMD, a molecular genetic deletion of part of the DMD gene will be found. A muscle biopsy may then be chosen to distinguish with confidence between the Duchenne and Becker types of $\mathrm{Xp} 21$ dystrophy. In the absence of a DMD gene deletion, the differential diagnosis is broader and the need for muscle biopsy is clearer. We recognise the inevitability of some delay in the diagnostic process, and have highlighted this positively as a time when parents can become part of the decision making process. Some parents have requested an immediate biopsy while others, although they have had DNA analysis, have opted to defer the biopsy. ${ }^{9}$

(3) To discuss patterns of future contact and assure parents of the availability of paediatric and other support. Mothers are more satisfied when they have the opportunity to ask questions of their informant and are free to contact them in the future. ${ }^{10}$ The fact that the paediatrician disclosing the results to the family is likely to be involved long term in the practical care of the child and family means that (s)he can give appropriate emphasis to the positive aspects of caring for a child with DMD - what there is to work for in caring for an affected boy, what he can gain from life, what the family and the professionals can achieve together. This may enable the paediatrician to adopt Brewin's 'third way' of giving bad news - helping the family to focus on the positive aspects of what can be achieved by and for the affected child. ${ }^{11}$

PHASE 3: FOLLOW UP-THE PROVISION OF ON-GOING SUPPORT

A disclosure is not an isolated event but needs to be seen in the context of a family's on-going life. It is important to identify a key worker at the primary health care level who has a positive relationship with the family; in practice it is often the health visitor. This person plays a vital role as 'gate keeper' to the family-to protect the family from an overload of health professionals, and to know the family well enough to know their current needs. The support the family receives will only be effective if the key worker herself receives support and information on the specialist services that are available. A strong link between the central newborn screening team and the primary health care team is vital, and this is facilitated by the pre-encounter meeting.

\section{The protocol in practice}

The protocol has now been in operation for more than four years, and 41 families have been interviewed ( 15 with a transient creatine kinase increase, 15 with a confirmed positive result, and 11 with a later diagnosis). They were all asked to rank their level of satisfaction with how the situation had been handled from $1=$ very poor to $5=$ excellent. Of the 41 families, 25 had the news broken using the protocol, the remaining 16 did not-either because the diagnosis was made after symptomatic presentation rather than newborn screening or because the protocol had not been introduced. A significant difference was found between the two groups with $88 \%$ of families in the protocol group saying that the process had been handled well or excellently, compared with $19 \%$ in the nonprotocol group. The average score for the protocol group was 4.3 , while the non-protocol group mean was $2.6 \quad\left(\chi^{2}=19.09, \mathrm{df}=4\right.$, $\mathrm{p}<0.01)$. If transient cases are omitted, then a significant difference between the two groups persists $\left(\chi^{2}=11.17, \mathrm{df}=4, \mathrm{p}<0.05\right)$. One mother praised the competent handling of the programme in these words: 'The way it was handled you could say nothing that it wasn't competent from beginning to end...'. Other mothers specifically commented on the speed of getting the venous blood result and the time, care, and concern shown by the doctor, all elements highlighted in the protocol. Thirteen of 15 families with a boy identified as having DMD through this programme are positive about newborn screening for DMD. One family regrets having had the test; their child was identified early in the programme before the protocol described here was developed. Furthermore, that family also had an older, but previously undiagnosed, affected boy as well as the child identified by the programme.

In March 1993 a questionnaire was sent to relevant health professionals in Wales, which showed that there had been a significant shift in attitude with $79 \%$ in favour of screening at that time while only $66 \%$ reported that they had 
been in favour in 1990 ( $n=426$ respondents). The main reason given for this change was their personal experience of the screening programme and its protocol in practice. The early indications from our research would indicate that the use of our protocol has increased levels of satisfaction in both families and health professionals.

\section{Conclusion}

While screening in the newborn period for DMD is still under evaluation, preliminary evidence indicates that the excessive trauma anticipated in making such a disclosure presymptomatically has been avoided as a result of implementing a strict protocol of disclosure and support, a feature already recognised by commentators. ${ }^{612}$ The protocol has been designed to address the issue of how the disclosure is managed and has four underlying principles:

(1) To maximise choice: At every stage from screen to diagnosis parental choice should be facilitated ensuring that they feel part of the decision making process.

(2) To maximise information: At every stage, beginning with the offer of the original test, parents should be provided with maximum unbiased information on which to base their decisions.

(3) To minimise delays in giving results: Time taken to plan practical details in advance can ensure that the family do not experience additional stress waiting for results.

(4) To facilitate on-going support: The preliminary meetings with the primary health care team and with the paediatrician provide information, focus attention on the disclosure, and facilitate on-going support for the family.
A strategy for breaking bad news that is family centred, rather than calibrated to the clinicians' own comfort levels, ${ }^{13}$ may be the key to reducing patient dissatisfaction. This protocol may explain why the newborn screening programme for DMD has been better accepted in practice than was initially anticipated in principle.

We would like to acknowledge the financial support received for this programme from the Muscular Dystrophy Group of Great Britain and the Wellcome Trust.

1 Bradley DM, Parsons EP, Clarke A. Preliminary experience with newborn screening for Duchenne muscular dystrophy. BMF 1993; 306: 357-60.

2 Gath A. Parental reactions to loss and disappointment: the diagnosis of Down's syndrome. Dev Med Child Neurol diagnosis of Down's

3 Cunningham CC, Morgan PA, McGucken RB. Down's syndrome: is dissatisfaction with disclosure of diagnosi inevitable ? Dev Med Child Neurol 1984; 26: 33-9.

4 Standing Conference of Voluntary Organisations for People with a Mental Handicap in Wales (SCOVO). Parents deserve better. A review report on early counselling in Wales. Cardiff: SCOVO, 1989.

5 SCOPE. Right from the start- looking at diagnosis and disclosure. London: SCOPE, 1994.

6 Statham H, Green J, Snowdon C. Mothers' consent to screening newborn babies for disease. $B M \mathcal{F}$ 1993; 306: 858-9

7 Philp R. Midwives and screening tests. Cardiff: University of Wales College of Medicine, 1993 (dissertation).

8 Edmund C. Unlinked anonymous HIV testing: a study of attitudes of midwives and mothers to testing. Cardiff: University of Wales College of Medicine, 1994 (dissertation).

9 Parsons EP, Clarke AJ, Bradley DM. 'They must have got it wrong': the experience of families identified by a newborn screening programme for Duchenne muscular dystrophy. In: Bloor M, Taraborrelli $P$, eds. Oualitative studies in health and medicine. Aldershot: Avebury, 1994.

10 Sloper P, Turner S. Determinants of parental satisfaction with disclosure of disability. Dev Med Child Neurol 1993; 35: 816-25.

11 Brewin TB. Three ways of giving bad news. Lancet 1991; 337: 1207-9.

12 Bowman JE. Screening newborn infants for Duchenne muscular dystrophy. BMF 1993; 306: 349.

13 Taylor KM. 'Telling bad news': physicians and the disclosure of undesirable information. Sociology of Health and Illness 1988; 10: 109-32. 